\title{
INCIDENCIA DE LA ECO-INNOVACIÓN EN LA COMPETITIVIDAD DEL SECTOR HOTELERO DE LA CIUDAD DE BOGOTÁ'.
}

\author{
Rubén Dario Díaz Mateus ${ }^{2}$ \\ Sofía Victoria Higuera Quintero ${ }^{3}$ \\ Julieth Paola Abadía Aguirre ${ }^{4}$
}

Recibido: 27 de abril de 2013

Aceptado: 12 de agosto de 2013

\begin{abstract}
"Salvaguardar el medio ambiente.... Es un principio rector de todo nuestro trabajo en el apoyo del desarrollo sostenible; es un componente esencial en la erradicación de la pobreza y uno de los cimientos de la paz"
\end{abstract}

Kofi Annan

\section{Resumen}

La población objeto de estudio está representada por los hoteles con sello ambiental colombiano SAC. La investigación tiene como objeto identificar la incidencia de la eco-innovación en la competitividad del sector hotelero de la ciudad de Bogotá, se siguió un proceso metodológico de orden deductivo-inductivo con el propósito de analizar las prácticas ambientales más representativas desarrolladas en el sector hotelero considerando el factor eco-innovador como elemento competitivo en la generación de beneficios de tipo económico y ambiental. En efecto, el modelo de turismo predominante en Bogotá es de negocios o metropolitano, por tanto, los avances innovadores están dados en la estructura de procesos, ésta implica nuevos métodos de producción o distribución, mejoras técnicas, equipos o software y por último innovación de servicio y organizacional.

Palabras clave: Innovación, eco-innovación, competitividad, hotel, Bogotá.

\footnotetext{
1 Esta investigación es el resultado de un proyecto de investigación desarrollado en el marco del semillero de investigación en Gestión Ambiental y Social de las organizaciones (GEAMSO) de la Universidad de La Salle; adscrito al Grupo de Investigación Desarrollo y Sociedad (DESUNISALLE).

2 Economista, Universidad Pedagógica de Colombia (UPTC). Candidato a doctor en Economía Aplicada de la Universidad de Santiago de Compostela (España). Maestría Economía Aplicada (DEA) Universidad de Santiago de Compostela (España). Especialista en planeación y gestión del Desarrollo territorial (UPTC). Docente de la Facultad de ciencias Administrativas y Contables, Universidad de La Salle. Líder de investigación grupo Desarrollo y Sociedad. rudiaz@unisalle.edu.co

${ }^{3}$ Estudiante de Administración de Empresas en la Universidad De La Salle, integrante del semillero de investigación en Gestión Ambiental y Social en las organizaciones (GEAMSO) Universidad de La Salle. Correo electrónico: shiguera01@unisalle.edu.com

${ }^{4}$ Estudiante de Administración de Empresas en la Universidad De La Salle, integrante del semillero de investigación en Gestión Ambiental y Social en las organizaciones (GEAMSO) Universidad de La Salle. Correo electrónico: paolaabadia@gmail.com
} 


\title{
INCIDENCE OF ECO-INNOVATION IN THE COMPETITIVENESS OF THE HOTEL INDUSTRY IN THE CITY OF BOGOTA.
}

\author{
"Safeguarding the environment it is a guiding principle of all our work in \\ support of sustainable development. Is an essential component in the \\ eradication of poverty and one of the foundations of peace" \\ Kofi Annan.
}

\begin{abstract}
The study population is represented by hotels with Colombian environmental seal (SAC). The research aims to identify the impact of eco-innovation in the competitiveness of the hotel industry in the city of Bogota, was followed by a methodological process of deductive-inductive in order to analyze the most representative environmental practices developed in the hotel sector considering eco-innovative factor as competitive element in generating economic benefits and environmental type. Indeed, the predominant model of tourism in Bogotá is business or metropolitan, therefore innovative developments are given in the process structure, this implies new methods of production or distribution, technical improvements, equipment or software and last service and organizational innovation.
\end{abstract}

Keywords: innovation, eco-innovation, competitiveness, hotel, Bogota.

\section{Introducción}

La ausencia de avances significativos en el campo de la administración es uno de los desafíos de nuestra época (Hammel \& Breen, 2008), el mundo demanda soluciones eficientes a un sin número de necesidades a nivel social, económico, ambiental y cultural, requiriendo el desarrollo de nuevos modelos administrativos que sean flexibles, competitivos, adaptables, colaborativos y acordes a las necesidades exigidas globalmente, destacando todas aquellas relacionadas con el medio ambiente.

Millán, Rosa, \& Villarreal (2009) mencionan en los sucesivos fenómenos que denotan el deterioro del medio ambiente derivado de la excesiva actividad humana hacen que expertos académicos, gobiernos y distintas organizaciones sociales, periódicamente manifiesten su preocupación y realicen propuestas para ubicar la protección medio ambiental en el centro de las discusiones.
La eco-innovación es una herramienta de gestión y estrategia, que responde a los retos presentes y futuros del desarrollo sostenible, implicando una rápida modificación de los procesos mentales e industriales (Fussler \& James, 1996), así, se puede generar la construcción de escenarios sujetos a un nuevo modelo de crecimiento económico «verde». La eco-innovación puede ser vista como una oportunidad de cambio transcendental para la sociedad y las organizaciones, que deberán ser cada vez más conscientes del impacto socio-ambiental que genera el desarrollo de su actividad económica.

La presente investigación tiene por objetivo identificar la incidencia de la eco-innovación en la competitividad del sector hotelero de la ciudad de Bogotá, a través de tres teorías importantes a seguir; la primera, corresponde a la eco-innovación desde la teoría de Peter James y Claude Fussler; en segundo lugar, la 
innovación desde la perspectiva del Manual de OSLO y finalmente la Competitividad bajo el enfoque del diamante competitivo y la matriz de estrategias genéricas de Michael Porter, lo anterior se logrará a través de la caracterización de la composición y singularidades actuales del sector, seguido de una descripción de los principales factores que conllevan a los hoteles a la ejecución de prácticas eco-innovadoras, lo que finalmente permitirá determinar el impacto de la eco-innovación en el sector hotelero de la ciudad de Bogotá en términos sociales, económico, ambientales y competitivos.

\section{Desarrollo}

La caracterización del concepto de la ecoinnovación nace del significado explícito de la innovación. Así, a nivel nacional, el concepto de innovación se define como el proceso mediante el cual la sociedad extrae del conocimiento beneficios económicos y sociales (Departamento Nacional de Planeación, 2007); posteriormente en el año 2011 esta institución, en el marco de la estrategia nacional de innovación, conceptualiza la innovación en la asimilación y explotación exitosa de una invención en la esfera económica y social, con el fin de aportar soluciones nuevas a los problemas nacionales y regionales, permitiendo responder a las necesidades de la sociedad y el sector público. El Plan Nacional de Desarrollo, 2010-2014, describe la innovación como la «introducción de un nuevo o significativamente mejorado, producto (bien o servicio), de un proceso, de un nuevo método de comercialización o de un nuevo método organizativo, en las prácticas internas de la empresa, la organización del lugar de trabajo o las relaciones exteriores» (p.56). Para el año 2012, dentro del marco de la Agenda Nacional de Competitividad conviene la innovación en la incorporación de conocimiento para agregar valor a los productos, procesos y de esta forma incrementar la productividad del país.
Por último, La Organización para la Cooperación y Desarrollo Económico (2005) la describe como «la implementación de un producto (bien o servicio) o proceso nuevo o con un alto grado de mejora, o un método de comercialización u organización nuevo aplicado a las prácticas de negocio, al lugar de trabajo o a las relaciones externas» (p. 33). Es fundamental mencionar el concepto de innovación según el Manual de Oslo al corresponder al documento directriz más utilizado en el mundo para conocer las actividades de innovación en las organizaciones donde la «concepción e implantación de cambios significativos en el producto, el proceso, el marketing o la organización de la empresa con el propósito de mejorar los resultados» (Manual de Oslo, 2005: 44).

En consecuencia, la Fundación Observatorio de Prospectiva Tecnológica Industria (OPTI) y Fundación (EOI) Escuela de Organización Industrial (2010), argumentan que el cambio de conciencia social conlleva a la creación de una nueva demanda, más sostenible y respetuosa con el medio ambiente, traducida en una serie de actuaciones públicas (Estado) y privadas (Empresas) interesadas en controlar, minimizar, corregir y prevenir los efectos adversos de la actividad económica sobre el medio ambiente, con racionalidad más eficiente de los recursos naturales.

Por lo tanto, la economía verde se ha configurado como un nuevo modelo de desarrollo económico basado en la economía ecológica y la sostenibilidad, contraponiéndose al modelo actual de economía negra, centrado en el uso del carbón, petróleo y otras fuentes de energía no renovables. Su principio fundamental se origina de la preocupación por la interdependencia entre las personas y los sistemas naturales, por el impacto de las actividades económicas en el cambio climático, involucrando organizaciones (privadas-públicas) y personas que la identifican como un elemento transversal en la sociedad. 
En este sentido, la eco-innovación es un concepto reciente, creado en 1996 por los ingenieros Claude Fussler y Peter James, basado en la confluencia de teorías económicas y de innovación enfocadas hacía la generación de productos y servicios que cumplieran con una característica específica, reducir significativamente el impacto nocivo sobre el medio ambiente y los altos índices de contaminación en el proceso de producción.

Asimismo, Carrillo, autor principal del libro: EcoInnovation: When sustainability and Competitiveness Shake Hands (2009), estructura un nuevo concepto que contribuye al desarrollo sostenible, reduciendo el impacto medioambiental y optimizando el uso de los recursos, desde dos perspectivas: la primera, desde la innovación, refiriéndose a la creación de productos y procesos que reducen el deterioro del medio ambiente; la segunda desde la perspectiva económica, buscando en las empresas un uso eficiente de los recursos naturales para mejorar el bienestar humano. Consecuentemente, el concepto de eco-innovación definido por Fussler y James en su libro eco-innovación integrando el medio ambiente en la empresa del futuro (1999), parte de la creación de «nuevos procesos y productos que deben incrementar el valor de los clientes y negocios pero con una reducción significativa del impacto sobre el medio ambiente y la contaminación» (p. 319).

Además, la Comisión Europea (2004), identifica la eco-innovación como todas las tecnologías y servicios que contribuyen a un mejor entorno; es decir, cualquier forma de innovación que busque un avance significativo y sostenible mediante la reducción del impacto ambiental y el logro eficiente en el uso de los recursos naturales y la energía. En efecto, la eco-innovación se puede llevar a cabo en cualquier etapa del ciclo de vida de un producto o servicio; este concepto implícitamente, esta relacionado con la eco-eficiencia y la eco-industria, cuyo objetivo es obtener un incremento en el valor de los bienes o servicios pero con un menor impacto ambiental, para lo cual, la idea principal es desvincular el crecimiento económico y la presión ambiental; de esta manera, la eco-eficiencia se encarga de medir el impacto ambiental de un producto o servicio; mientras que la eco-industria incorpora el uso de tecnologías más limpias, productos y servicios que minimicen la contaminación y el uso de los recursos.

\section{Definiciones de innovación, eco-innovación y competitividad}

\section{Innovación}

Fuente

BANCOLDEX ${ }^{5}$

Es la creación de valor a través de la transformación de ideas o conocimientos, en productos (bienes o servicios) nuevos o significativamente mejorados.
Boston Consulting Group
Define innovación no únicamente como el desarrollo de nuevos productos, sino también se encuentra involucrada en el modelo de negocios, estructura empresarial, cadena de valor, procesos propietarios, canales, servicios, marca y la experiencia del cliente.

DNP6 $^{6}$

La innovación, es la asimilación y explotación exitosa de una invención en la esfera económica y social, con el fin de aportar soluciones nuevas a los problemas nacionales y regionales, permitiendo responder a las necesidades de la sociedad y el sector público.

${ }^{5}$ Banco de Desarrollo Empresarial y Comercio Exterior de Colombia conocido por sus siglas en español como BANCOLDEX.

${ }^{6}$ Departamento Nacional de Planeación por sus siglas en español DNP. 
Freedman

La innovación es el proceso de integración de la tecnología existente y los inventos para crear o mejorar un producto, un proceso o un sistema. Innovación en un sentido económico consiste en la consolidación de un nuevo producto, proceso, o sistema mejorado.

Goodman

Es el conjunto de actividades inscritas en un determinado periodo de tiempo y lugar que conlleva a la introducción con éxito en el mercado.

Manual de Oslo

Es la puesta en marcha de un producto, bien, servicio, de un proceso nuevo o mejorado, y de un nuevo método de comercialización u organización de la empresa y relaciones existentes.

«Innovar no sólo significa desarrollar nuevos productos y transformar los productos existentes, la innovación consiste en crear nuevas formas de producir, $\mathrm{MCIT}^{7}$ entregar, comercializar y vender, logrando en última instancia generar valor agregado a través de toda la cadena productiva».

«La Innovación es el elemento clave de la competitividad». La

Michael Porter Competitividad de una nación depende de la capacidad de su industria para innovar y mejorar. Las empresas consiguen ventajas competitivas si consiguen innovar.

La innovación es la herramienta específica de los empresarios innovadores;

Peter Drucker el medio por el cual se puede explotar el cambio como una oportunidad para un negocio diferente. Es la acción de dotar a los recursos con una nueva capacidad de producir riqueza.

Es la introducción en el mercado de un nuevo bien, método de

Schumpeter producción aun no experimentado; la adopción de una nueva fuente de suministro de materias primas o semielaborados, o la implantación de una nueva estructura en un mercado.

Determina que la Innovación es un factor clave para medir el crecimiento de la productividad, por lo tanto comprender su origen y sus patrones es fundamental para desarrollar mejores políticas.

Unión Europea Las dimensiones de la innovación son Recursos Humanos, Financiación y apoyo, Inversiones de empresa, Vínculos e iniciativa empresarial, Rendimientos, Innovadores y Efectos económicos.

Entiende la innovación como uno de sus doce pilares que configuran la comWEF $^{8}$ petitividad, definida como «el conjunto de instituciones, políticas y factores que determinan el nivel de productividad de un país».

\begin{tabular}{ll} 
Fuente & \multicolumn{1}{c}{$\begin{array}{c}\text { Eco-Innovación } \\
\text { Definición }\end{array}$} \\
& $\begin{array}{l}\text { Basado en la confluencia de teorías económicas y de la innovación. Ellos se } \\
\text { basan en la premisa de satisfacción al cliente, enfocada hacia la generación } \\
\text { de productos y servicios con una característica especial; reducir significa- }\end{array}$ \\
tivamente el impacto nocivo sobre el medio ambiente y los altos índices de \\
Peter James \\
(1996) & $\begin{array}{l}\text { contaminación en el proceso de producción. } \\
\text { «Eco-innovación es el proceso de desarrollo de nuevos productos, procesos } \\
\text { o servicios que proporcionan valor al negocio y cliente, al mismo tiempo en } \\
\text { que disminuyen el impacto ambiental». }\end{array}$
\end{tabular}

Klemmer et al, (1999).

Eco-innovaciones son todas las medidas de las partes interesadas (empresas, políticos, sindicatos, asociaciones, iglesias, hogares privados) que desarrollan nuevas ideas, comportamientos, productos y procesos que contribuyen a la reducción de las cargas ambientales. 
Comisión Europea (2004)

Organización para la Cooperación y el Desarrollo Económicos (OCDE , 2008)

Europa Innova (2006).

(2006) (2007)

Comisión Europea

Technopolis group (2008).
Eco-innovación son todas las tecnologías y servicios que contribuyen a un mejor entorno.

Conjunto de actividades que producen bienes y servicios para medir, prevenir, limitar, minimizar o corregir daños ambientales en temas de agua, aire y polución así como problemas relacionados con los residuos, contaminación acústica y ecosistemas. Esto incluye las tecnologías más limpias, los productos y los servicios que reducen riesgo ambiental y minimizan la contaminación y el uso de recursos.

«Eco-innovación es la creación de nuevos productos y precios competitivos, procesos, sistemas, servicios y procedimientos diseñados para satisfacer las necesidades humanas y proporcionar una mejor calidad de vida para todos, con un uso mínimo del ciclo de vida de los recursos naturales (materias incluyendo la energía, y el área de superficie) por unidad de producto, y una liberación mínima de sustancias tóxicas».

Eco-innovación es cualquier forma de innovación que persiga un avance significativo y demostrable respecto al objetivo del desarrollo sostenible, a través de la reducción de los impactos sobre el medio ambiente y el logro de un uso más eficiente y responsable de los recursos, incluida la energía.

«Eco-innovación es cualquier forma de innovación que persiga un avance significativo y demostrable respecto del objetivo del desarrollo sostenible, a través de la reducción de los impactos sobre el medio ambiente y lograr un uso más eficiente y responsable de los recursos naturales, incluida la energía».

Eco-innovación es un tipo de innovación sostenible como un proceso en el que las consideraciones de sostenibilidad (ambiental, social, económica) están integrados en sistemas de la empresa de generación de ideas a través de la investigación y el desarrollo $(I+D)$ y la comercialización. Esto se aplica a los productos, servicios y tecnologías, así como de nuevos negocios y modelos de organización.

Es la creación de una novedad competitiva en la producción de bienes, servicios, sistemas y procedimientos, diseñados para satisfacer las necesidades humanas y proporcionar una mejor calidad de vida, con un mínimo uso de los recursos naturales (tanto materiales como energéticos) por unidad de producción en todo el ciclo de vida y un vertimiento mínimo de sustancias tóxicas.

Kemp, R. and P.

Pearson

Eco-innovación es la producción, asimilación o la explotación de un produc(2008). to, proceso de producción, servicio, método de gestión o de negocio que es una novedad en la organización (desarrollada o adoptada), y que implica a lo largo de su ciclo de vida una reducción de los riesgos ambientales, la contaminación y otros impactos negativos del uso de recursos (incluyendo la energía) en comparación con las correspondientes alternativas.

OCDE $^{9}$

Eco-innovación es la creación o mejoramiento de productos, procesos, méto(2008). dos de marketing, estructuras organizacionales o acuerdos institucionales que producen mejoras ambientales comparadas con sus alternativas relevantes.

Huppes, Kleijn, Huele, Ekins, Shaw,Schaltegger Eco-innovación es un cambio en las actividades económicas que supone una y Esders (2008) mejora tanto en la actuación económica como ambiental para la sociedad. 


\begin{abstract}
Expresa la Eco-Innovación como un nuevo desafío para las empresas y las políticas públicas, definida como todo tipo de innovación que contribuye al Carrillo desarrollo sostenible, reduciendo el impacto medioambiental y optimizando (2011) el uso de los recursos; puesto que tarde o temprano, el daño medioambiental tendrá un impacto negativo en el sistema económico, y de forma inevitable habrá que pagar el costo. «La eco-innovación ofrece la posibilidad de reducir costos y generar beneficios, público- privado, que lo compensen».
\end{abstract}

\begin{tabular}{ll} 
Fuente & \multicolumn{1}{c}{ Competitividad } \\
\hline $\begin{array}{l}\text { European Manage- } \\
\text { ment Forum } \\
(1998)\end{array}$ & $\begin{array}{l}\text { Una medida de la capacidad inmediata y futura de los industriales de dise- } \\
\text { ñar, producir y vender bienes cuyos atributos en términos de precios y más } \\
\text { allá de los precios se combinan para formar un paquete más atractivo que el } \\
\text { de productos similares ofrecidos por los competidores: el juez final es enton- } \\
\text { ces el mercado. }\end{array}$
\end{tabular}

Haguenauer (1989)

La capacidad de una industria (o empresa) de producir bienes con patrones de calidad específicos, requeridos por mercados determinados, utilizando recursos en niveles iguales o inferiores a los que prevalecen en industrias semejantes en el resto del mundo, durante un cierto período de tiempo.

Buckley y otros (1988)

OCDE

(1996)

Porter

(1990)
Tanto la eficiencia (alcanzar las metas al menor costo posible) como la efectividad (alcanzar los objetivos correctos). Es la elección de las metas a nivel industria la que es crucial. La competitividad incluye tanto los fines como los medios utilizados para alcanzar esos fines.

La habilidad de las firmas, industrias, regiones, naciones o regiones supranacionales de generar altos niveles de empleo y de ingresos de los factores, mientras están expuestas a la competencia internacional.

La capacidad para sostener e incrementar la participación en los mercados internacionales, con una elevación paralela del nivel de vida de la población. El único camino sólido para lograr esto se basa en el aumento de la productividad.

Fuente: elaboración Propia basados en los autores referidos en cada ítem.

\section{Desarrollos investigativos de la eco-innovación}

Desde la aparición del concepto de Responsabilidad Social Empresarial (RSE) en el siglo $\mathrm{XX}$, las organizaciones tienen entre sus tareas más importantes identificar mediante una contribución activa y voluntaria el mejoramiento social, económico y ambiental de sus entornos (Universidad de Burgos, 2010), por tal razón, la literatura actual y la investigación se han centrado en la generación de prácticas y estrategias adaptables en las organizaciones y a los administradores con el fin de solucionar los problemas ambientales. En consecuencia, Griffiths y Haigh (2004) presentan un estudio que explora un programa de innovaciones organizacionales destinadas a mejorar las prácticas ambientales, éste adoptado seis empresas industriales, procesadoras del sector de cárnicos en Australia, analizando un total de seis empresas que hicieron uso de un programa y equipos basados en la resolución de problemas ambientales, sin embargo, sólo tres lograron institucionalizar las prácticas propuestas mediante el programa.

Por otra parte, Hellström (2007) analizó la estructura de los conceptos eco-innovadores, mediante el análisis de sus diferentes dimensiones en el medio ambiente y desde la perspectiva de las teorías existentes de la innovación, con el fin de establecer estructuras dominantes de estas innovaciones y las debilidades actuales. Para tal efecto, se desarrolló una investigación en la que se analizaron 105 conceptos de riesgo, 
dentro de los cuales se consideró una muestra derivada de las contribuciones ganadoras de un concurso de innovación. Como resultado, se observó un sesgo hacia las innovaciones de procesos graduales, pero se destacaron características interesantes de arquitectura radical en los modos de eco-innovación, por ejemplo, la tendencia a combinar hardware y software a través de la combinación de diversas tecnologías.

Otros estudios sobre eco-innovación han demostrado la importancia de la investigación en Eco-diseño, como factor crítico de éxito. En efecto, Santolariaa (2011) indagó acerca del Eco-diseño en las empresas de innovación, con el objetivo de contribuir al conocimiento del diseño ecológico y la sostenibilidad en relación a las empresas de innovación impulsada. Por ende, este trabajo se dividió en tres partes, la primera verifica la percepción y el conocimiento del eco-diseño por parte de profesionales de empresas innovadoras; la segunda clasificó aquellas que se dedican al diseño ecológico mediante la integración de estrategias basadas en la sostenibilidad aplicada a los planes estratégicos y de producción, por último, se dio origen a la creación de un escenario futuro que le permitiera generar predicciones acerca de lo que sucederá con las empresas en materia de innovación y eco-diseño. El estudio demostró que la sostenibilidad es un factor esencial para la innovación y que las respuestas tienen características puntuales en cuanto a tamaño de la empresa, actividad o cargo del entrevistado.

Investigaciones más recientes como las elaboradas por el Observatorio de Eco-innovación ${ }^{1}$ se han preocupado por el desarrollo de marcos económicos-ecológicos privilegiando el análi-

\footnotetext{
${ }^{10}$ El observatorio de eco-innovación Hace parte del Programa de competitividad e innovación propuesto en el año 2007 por la Comisión Europea, con el fin de actuar como un recurso de conocimientos sobre eco-innovación, esta red recopila y difunde análisis esenciales y actualizados sobre tecnologías medioambientales y de mercados de eco-innovación, Incorporando análisis estadísticos país por país y mercado por mercado proporciona una plataforma para la recolección y el análisis estructurado de una amplia gama de información sobre eco-innovación, recolectada de toda la Unión Europea y las regiones económicas clave en todo el mundo. Para mayor información visite el sitio web: http://www.eco-innovation.eu/
}

sis de la eco-innovación desde perspectivas interdisciplinares, tal es el caso de las tecnologías verdes o «Green IT»² y sus aplicaciones, para tal efecto, es necesario formular preguntas como ¿Qué se sabe sobre tecnologías verdes?, ¿considera que los edificios inteligentes, uso eficiente de las redes de energía y transporte son soluciones suficientes para el fortalecimiento de una economía ecológica? y, en caso afirmativo, de qué manera?, este tipo de preguntas son formuladas en la investigación llevada a cabo por Faucheuxa y Nicolaïb (2011) en donde se analiza el impacto económico, social y ambiental de las IT. El estudio se centran en la dimensión administrativa de la teoría eco-innovadora, al presentar uno de los rasgos distintivos más importantes de las aplicaciones verdes, tal es el caso de la organización colectiva de la innovación, identificada como una tipología de la innovación ecológica que busca conciliar el desarrollo de tecnologías verdes con el crecimiento verde, centrado esencialmente en cuatro tipos de cambios hacia el desarrollo sostenible: la innovación tecnológica, social, institucional y organizacional.

Finalmente, El observatorio de eco-innovación en su informe Closing de eco-innovations GAP (2012) se centró en el estudio de los beneficios económicos de la eco-innovación; basados en el último Informe Anual del Observatorio ${ }^{3}$, en el que se analizó un caso ambiental para la ampliación e intensificación de actividades eco-innovadoras en la Unión Europea. A lo largo del documento, se considera la eco-innovación en una oportunidad para los programas económicos y ambientales se apoyen incondicionalmente en las empresas europeas es la clave para lograr un cambio sistémico de toda la economía. Lo anterior implica tanto la reducción del consumo absoluto de recursos naturales como la mejora

\footnotetext{
${ }^{11}$ Green Computing también conocido como Green IT o traducido al español como Tecnologías Verdes se refiere al uso eficiente de los recursos computacionales minimizando el impacto ambiental, maximizando su viabilidad económica y asegurando deberes sociales

${ }^{12}$ El Desafío Eco-innovación: Rutas hacia una Europa eficiente en recursos (2011).
} 
de la competitividad de las empresas europeas. Se concluyó que existe una diferencia entre el potencial eco-innovador y el estado actual de la actividad eco-innovadora en la Unión Europea. Este informe proporcionó evidencia sobre el alcance significativo de los beneficios económicos derivados de la eco-innovación empresarial.

\section{Metodología}

Este análisis se debe considerar como un estudio experiencial y de observación, dentro de un contexto eco-innovador, con miras a la identificación de prácticas amigables con el medio ambiente en los hoteles de la ciudad de Bogotá, Colombia. La muestra de hoteles objeto de estudio ha sido elegida por conveniencia (hoteles con el sello ambiental colombiano SAC), la herramientas de recolección de información primaria será el cuestionario adaptado a tres tipos de actores, los cargos administrativos, operativos y a los clientes de los hoteles.

En esta investigación se utilizaron dos tipos de métodos, inicialmente por el método deductivo el cual brindará una perspectiva generalizada para señalar ciertas teorías verdaderas inmersas en un situación general, en este caso se parte de la teoría eco-innovadora como verdad universal, según Méndez (2006), en este método «la teoría general acerca de un fenómeno o situación, se explican hechos o situaciones particulares» (p. 240), por otro lado se utilizó el método inductivo el cual inició con una observación basada en el análisis de las actividades amigables con el medio ambiente, con el objeto de identificar cuál de ellas se puede definir como eco-innovadoras para determinar cuál es el impacto de estas prácticas en la competitividad de los hoteles, desde tres perspectivas en particular, la administrativa, la del cliente interno (funcionarios) y clientes externos (huéspedes).

Este proceso de conocimiento como lo indica Méndez (2009), «es fundamentalmente la inducción, en donde a pesar de tener como referencia premisas verdaderas, se pudo llegar a conclusiones cuyo contenido fue más amplio que el de las premisas en las cuales inicia el análisis de su problema de investigación» (p.239). Con respecto de las fuentes de información, se estableció dividir las fuentes primarias de información en tres cuestionarios de preguntas cerradas y opción múltiple, dirigidos a los cargos administrativos, operativos de los hoteles objeto de estudio, un tercer cuestionario fue elaborado para los clientes de los hoteles; entre las fuentes de información secundarias se encuentran; libros, investigaciones similares, entre otros.

La muestra final de los hoteles objeto de estudio conformada por 8 hoteles categorizados como 5 estrellas, 2 hoteles categorizados como tres estrellas y 11 hoteles certificados por el Sello Ambiental Colombiano (ver cuadro No 1). En tanto, se aplicaron dos tipos de cuestionarios el primero para cargos administrativos, el segundo, para cargos operativos. El primero está compuesto por cuatro secciones, 57 preguntas en total, dentro de las cuales 28 son preguntas cerradas y 29 de opción múltiple. El segundo cuestionario dirigido a los cargos operativos tiene un total de 78 preguntas de las cuales 44 son preguntas cerradas y 34 de opción múltiple; con este cuestionario se recolectó información de los aspectos socioeconómicos, las prácticas ambientales que desenvuelven (únicamente se tuvieron en cuenta las relacionadas con el agua, la energía y manejo de residuos), posteriormente se obtuvo información acerca de la percepción de los encuestados sobre la innovación y la eco- innovación (ver cuadro No 2). 
Cuadro No 1. Muestra final de hoteles objeto de estudio

\begin{tabular}{|c|c|c|}
\hline \multicolumn{3}{|c|}{$\begin{array}{c}\text { Hoteles certificados por el Sello } \\
\text { Ambiental Colombiano }\end{array}$} \\
\hline 5 estrellas & 3 estrellas & No tienen estrellas \\
\hline Hotel Bogotá Plaza & $\begin{array}{l}\text { Hotel Centro } \\
\text { Internacional }\end{array}$ & Aparta Hotel Chico 93 \\
\hline Hotel De La Ópera & Hotel el Campín & GHL Hotel Los Héroes \\
\hline Hotel Morrison & & Hotel Embassy Suites \\
\hline Hotel Portón Bogotá & & Hotel Grand Hause \\
\hline Hotel Dann Carlton Bogotá & & Hotel Hamilton Court \\
\hline Hotel Sheraton Bogotá & & Hotel Las Terrazas \\
\hline Santafé Boutique Hotel & & Hotel Parque 97 Suites - \\
\hline \multirow[t]{4}{*}{ Suites 101 Park House S.A. } & & Hotel Retiro 84 \\
\hline & & Hotel San Pablo \\
\hline & & Hotel Estelar Windsor Hause \\
\hline & & Teusaquillo Boutique Hotel \\
\hline Total 5 estrellas $=8$ & Total 3 estrellas $=2$ & Total $S A C=11$ \\
\hline Total: 21 Hoteles & & \\
\hline
\end{tabular}

Fuente: Elaboración Propia

Cuadro No 2. Rangos de las preguntas según el tipo de cuestionario
Cuestionario cargos
Cuestionario cargos
Cuestionario
administrativos operativos para clientes

Aspectos socio económicos

2. Generalidades de las prácticas ambientales

1.1 Agua

1.2 Energía

1.3 Manejo de residuos
1. Generalidades acerca de los motivos de viaje y selección del hotel.
Generalidades de las prácticas ambientales
2. Generalidades de las prácticas ambientales, información y beneficios percibidos.

\section{Acerca de la innovación \\ 2. Acerca de la innovación \\ 3. Acerca de la innovación}

4. Acerca de la ecoinnovación

4. Acerca de la eco- innovación

Fuente: Elaboración Propia 


\section{Resultados}

El desarrollo de la actividad hotelera en la ciudad de Bogotá es significativo dentro del conjunto de actividades que más aportan a la economía local y nacional; con respecto al número total de hoteles que se encuentran en la ciudad el porcentaje de hoteles posee una certificación ambiental es de 10\% (Cotelco y Fedesarrollo, 2010). De otra parte se puede concluir que la muestra a nivel general presenta una noción errónea acerca del impacto que genera la actividad hotelera, afirman en un $52 \%$ que la prestación de servicios no genera impactos al medio ambiente, ya sea por desconocimiento o desinterés los empresarios deberían ser más conscientes de las consecuencias ambientales y sociales que trae no hacer nada al respecto.

\section{Caracterización, composición y singularidades del sector hotelero objeto de estudio}

- A nivel general se percibe la existencia de un aumento en el uso de prácticas ambientales; en consecuencia, se genera una tendencia de mejoramiento en el nivel de desempeño organizacional de los hoteles. Siguiendo la tendencia gana-gana de Porter y Van der Linde (1995).

- Se observa que existe aceptación al pensar que es posible obtener un mejor nivel de desempeño en función de las prácticas ambientales, pero se destaca la falta de personas capacitadas, y competentes para desarrollar y demostrar la viabilidad de proyectos ambientales.

- El $95 \%$ de los hoteles encuestados demostraron tener en su mayoría prácticas ambientales básicas como el uso de bombillos ahorradores de energía, políticas para el cambio en la ropa de cama, uso de grifería ahorradora de agua, procesos de reciclaje entre otros.
- $\quad$ El $78 \%$ de los hoteles considera en su visión, misión y objetivos organizacionales la preocupación por el medio ambiente, pero muchas veces en la práctica no se hace lo suficiente para contribuir al cuidado ambiental, se puntualiza en que este objetivo no es alcanzable sin la concientización, compromiso y colaboración de todas las personas involucradas en el hotel.

- El ámbito que más preocupó a los directivos de los hoteles fue el aspecto económico, se evidencia un interés constante por el tema económico frente al tema ambiental.

- Gran parte de las personas encuestadas lograron identificar las actividades que más impactan el medio ambiente considerando los servicios de cocina y lavandería como las áreas que más consumen agua en el hotel.

- Aunque los hoteles cuentan con políticas de sostenibilidad, políticas de responsabilidad social, certificaciones ambientales y manuales de buenas prácticas ambientales; se resalta que ninguno de los instrumentos anteriormente mencionados pueden ser eficientes sin el compromiso, concientización y colaboración de cada persona al servicio del hotel.

- En el $100 \%$ de los casos, los códigos de conducta, los manuales de buenas prácticas ambientales, las eco-etiquetas, los sellos ambientales, los sistemas de gestión ambiental e indicadores, son de acogimiento voluntario y sirven de base fundamental para el desarrollo de la eco-innovación al interior de las organizaciones.

- Más de la mitad de los hoteles consideran que la certificación ambiental trae beneficios a la organización, en consecuencia este proceso de certificación hace que el 
hotel cambie algunos comportamientos para cumplir con algunos parámetros, por ejemplo se observó por parte del hotel; la compra de productos biodegradables o menos nocivos al medio ambiente, políticas para el cambio de la ropa de cama, programas de reciclaje y publicidad en diferentes áreas del hotel con información relacionada al cuidado y preservación del medio ambiente.

- El $83 \%$ de los hoteles si conoce las normas obligatorias a las que debe acogerse respecto al tema ambiental; en consecuencia se observó una incoherencia respecto a la teoría y la aplicación de la norma ISO 14001, ya que dentro de la teoría se requiere demasiados registros de información de forma escrita lo que contribuye al uso exagerado de papel. Se sugiere encontrar otros medios para el registro de esta información, puede ser por vía electrónica.

- La totalidad de los hoteles encuestados manifestaron que dentro de sus estándares de selección de proveedores se encuentran aquellos que se preocupan por el medio ambiente y dentro de sus catálogos de productos ofertan productos biodegradables, naturales o amigables ambientalmente.

- Adicionalmente se detectó la falta de personal capacitado para llevar a cabo actividades y tareas ambientales.

- La totalidad de los hoteles visitados dispone de avisos con información ambiental para el huésped en lugares visibles como por ejemplo alcoba, baños, zonas comunes, entre otros; esto resalta el compromiso del hotel con el tema y a la vez le recuerda constantemente al cliente el cuidado y buen uso de los recursos naturales.

- En la mayoría de los hoteles objeto de estudio no se percibió un departamento de ges- tión ambiental, la causa principal según sus declaraciones se debe al tipo de turismo que predomina en la ciudad, es decir turismo de negocios o metropolitano; esto ha llevado a que los hoteles deleguen tareas de cuestiones ambientales a otros departamentos tales como de mantenimiento o de calidad;

- El $89 \%$ de los hoteles dispone de un departamento de innovación, y el 67\% cuentan con espacios para la generación y participación de ideas innovadoras, lo cual es fundamental para iniciar procesos de ecoinnovación e involucrar participativamente a los empleados y clientes.

- El $89 \%$ de las organizaciones creen que la innovación es un factor indispensable para lograr la competitividad del hotel, reconociendo la innovación como fuente de la ecoinnovación, se percibió la participación de los empleados administrativos y operativos en espacios para la generación de ideas innovadoras como mecanismo de solución a problemas generales del hotel.

- En los hoteles encuestados se evidenció que el tipo de innovación más utilizado es la innovación de procesos implicando en este tipo de innovación un nuevo método de producción o distribución, mejoras en técnicas, equipos o software; seguidamente se encuentra la práctica de la innovación de producto y por último la innovación organizacional.

- Es importante tener claro que la idea genera un invento, el invento es innovación; por lo tanto innovación es igual a crear y modificar, pero esto sólo será efectivo si se genera un proceso de comercialización y adopción de la misma en el mercado.

- Más de la mitad de los empleados con cargos administrativos, operativos y los clientes 
encuestados dicen no haber escuchado con anterioridad el término de eco-innovación, lo que conlleva a pensar que éste término en Colombia es un nuevo y despertó el interés de los encuestados.

- Es necesario que la eco-innovación se mida por producto, proceso, equipo y a nivel organizacional, ya que estos son los diferentes tipos de innovación que existen según el manual de Oslo y aplican para el proceso de eco-innovación, por ejemplo los productos eco-innovadores deben ser considerados desde la perspectiva de producto, proceso, mercadotecnia y uso.

- La eco-innovación se deriva de la innovación y abarca diferentes tipos de innovación por lo tanto dependiendo su grado podemos catalogar que para un hotel puede presentarse eco-innovación radical para otro hotel puede ser eco-innovación incremental, para un hotel puede ser eco-innovación aplicada a producto o proceso, para otro hotel puede ser eco-innovación organizacional.

- El $61 \%$ de los hoteles encuestados considera que el medio ambiente no interfiere en la competitividad de la organización, lo cual refleja que los empleados ven el medio ambiente como un factor independiente que no afecta la competitividad.

\section{Principales factores que conllevan al sector hotelero objeto de estudio a las ejecución de practicas eco-innovadoras}

- Gran parte de los hoteles analizados, considera la eco-innovación como un elemento clave de éxito para el hotel y manifiestan que la implementación de prácticas ecoinnovadoras generarán beneficios de tipo económico y ambiental.

- Por otra parte el diamante competitivo y la matriz de estrategias genéricas de Porter permitió identificar que la eco-innovación es un factor diferenciador que genera una rivalidad alta entre los competidores existentes, quien más practicas gestiona, mejores modelos de ahorro maneja, lo que además le permite cautivar mayor flujo de clientes internacionales.

- La amenaza de nuevos aspirantes evidencia que no hay gran dificultad para entrar, pues la curva de aprendizaje necesaria para ser competitivos es baja; pero, la diferenciación en el mercado a través de los precios es alta. Por otro lado, vale la pena resaltar que la inversión necesaria para ingresar y presentar una alternativa con alto grado de calidad e innovación es muy elevada, pero también es cierto que un gran conjunto de acciones enfocadas en la eco-innovación no requieren elevadas sumas de dinero, por el contrario demandan tiempo y la planeación de procesos de control y evaluación eficientes.

Se evidencia un bajo poder de negociación por parte de los proveedores, ya que ellos están en un alto grado dispuestos a satisfacer los requerimientos de los hoteles en cuanto a los tipos de productos que demandan para satisfacer las necesidades de sus huéspedes, quienes en un alto porcentaje se encuentra dispuestos a pagar tarifas más altas siempre y cuando se sientan seguros de su aporte con el medio ambiente.

- La eco-innovación es concebida como una ventaja estratégica para las organizaciones a través de estrategias de diferenciación o de liderazgo en costos.

- La exclusividad: permite ofrecer un servicio de hospedaje percibido como único a través de la generación de beneficios tangibles e intangibles, mediante la implementación de la eco-innovación en 
las estrategias de marketing y ventas, con miras al fortalecimiento de la lealtad de los clientes hacia la marca y a la menor sensibilidad al precio resultante.

- Liderazgo en costos: a través de la implementación de acciones eco-innovadoras los hoteles tienen costos reales más bajos que sus competidores (ahorros de más del $40 \%$ en consumo de agua y luz).

\section{Discusión}

Según los resultados de la encuesta aplicada se puede interpretar lo siguiente:

El cien por ciento de los hoteles encuestados consideraron la eco-innovación como un factor clave de éxito organizacional; aunque afirmaron que el medio ambiente no interfiere directamente en su competitividad. Lo anterior se sustentó en tres grandes afirmaciones, la primera, el factor impulsor para la práctica de la eco-innovación es claramente el beneficio económico, ubicando el bienestar ambiental en un segundo plano, en efecto, se consideraron como significativos, pero no son la prioritarios. Segundo, el tipo de turismo que se maneja en la ciudad (negocios) y el tercero derivado de la notoria ausencia de herramientas que permitan la identificación de impactos y de herramientas para su medición.

En consecuencia, la eco-innovación no se consideró como una estrategia de gestión objetiva por el sector empresarial hotelero, debido a la ausencia de información. En efecto, son pocos los empresarios que manifestaron su interés en la eco-innovación vista como una ventaja competitiva. Se desconoce en la mayoría de los casos la capacidad de gestionar grandes cambios a través de su implantación en el horizonte estratégico gerencial, pues añade valor a través de la búsqueda eficiente de soluciones a las necesidades y oportunidades futuras del mercado, mediante la generación de ideas novedosas dentro de un contexto de análisis de las necesidades de los clientes y del entorno a través de la innovación, esto permitiría a la organización obtener una posición estratégica en el mercado, mediante la diferenciación en sus servicios o en la eficiencia de sus costos.

Aunque se observó un incremento en el uso de las prácticas ambientales en la muestra objeto de estudio, éstos se catalogaron como Eco-innovadores pasivos debido a que no poseen estrategias específicas de innovación ambiental. Además se destaca la ausencia de un departamento dedicado a la gestión ambiental del hotel; además, la cantidad de personal capacitado para llevar a cabo pequeñas actividades y tareas de carácter ambiental es casi nulo. Por otra parte es importante destacar que el tamaño del hotel influyó en la facilidad de implantación y velocidad de acogimiento de los proyectos ambientales.

\section{Recomendaciones}

Dentro de las recomendaciones a los hoteles de la ciudad de Bogotá basados en la información obtenida y expresada a lo largo del presente artículo, se encuentran:

La creación de un departamento de gestión ambiental con personal capacitado e idóneo para desempeñar los proyectos ambientales propuestos.

Estudiar constantemente casos eco-innovadores exitosos, especialmente si se desarrollan en contextos económicos, sociales y ambientales similares al colombiano con el fin de establecer comparaciones constantes que permitan al hotel estar a la vanguardia en este tema.

Realizar programas de concientización ambiental medibles a través de indicadores, para ser dirigidos a los clientes internos y externos del hotel con el fin de dar a conocer la eco-innovación como una herramienta de gestión clave para la consecución de los objetivos concernientes con la Responsabilidad Social Empresarial (RSE). 
Establecer procesos de vigilancia tecnológica, benchmarking e investigaciones con miras a la identificación de las tendencias de mercado con respecto de los procesos innovadores y ecoinnovadores en el sector hotelero y afines.

\section{Conclusiones}

A través del diamante competitivo de Porter se obtuvo un análisis que permite identificar aspectos importantes para entender el movimiento de las fuerzas dentro del sector; en consecuencia, se destaca la rivalidad entre los competidores y la amenaza de los mismos es alta, por tanto, las barreras de entrada en este mercado son sencillas. Por otra parte, la amenaza de servicios sustitutos es media y no se considera peligrosa, debido a que el tipo de turismo que atiende la ciudad de Bogotá es de negocios, y los empresarios se inclinan ampliamente por hospedarse en hoteles reconocidos. Seguidamente se estableció que el poder de negociación por parte de los clientes y proveedores con los hoteles es bajo, esto se evidencia en la débil relación que existe entre los actores, los clientes no tienen una influencia significativa sobre las decisiones del hotel, de la misma forma ocurre con los proveedores.

De otro lado se presentó a la eco-innovación a través de la matriz de estrategias genéricas de Michael Porter, como una ventaja y objetivo estratégico, con el fin de reconocerla como herramienta de competitividad. En efecto, la eco-innovación vista como ventaja estratégica permite ofrecer a los hoteles un servicio diferenciado, a través del diseño de planes y ejecución de actividades que envuelvan a los clientes, haciéndolos sentir únicos, importantes y valiosos en el proceso de reducción de los impactos al medio ambiente en el proceso de prestación del servicio apoyado en la estrategia de conquistar un creciente mercado reconocido como «eco» 0 «verde».
Otra estrategia que puede elegir los hoteles está relacionado con el liderazgo en costos, mediante la revisión de procesos, rediseño, planeación e implementación de nuevas y mejoradas estrategias basadas en prácticas eco-innovadoras; está demostrado que a través de estas estrategias los hoteles pueden establecer topes que permitan identificar claramente las reducciones generadas. Lo anterior tiene como objetivo poder ofrecer al mercado precios más competitivos, que permitan al hotel hacer uso de estos ahorros o excedentes de capital en procesos que favorezcan la innovación.

El turismo es un sector intensivo en recursos y que deja una importante huella en el medio ambiente (Hunter y Shaw, 2007). Por ende el mantenimiento de la dinámica turística sólo se puede asegurar si se diseñan y aplican instrumentos destinados a incorporar procesos de eco-innovación tecnológico en el sector hotelero.

En consecuencia el mercado, la competencia, la globalización, el eco-cliente y las facilidades de viajar actualmente están haciendo surgir un nuevo modelo turístico. La adaptación del sector hotelero al nuevo modelo turístico pasa por un modelo más competitivo y sostenible ecológicamente. El creciente interés de la industria turística por la acreditación ambiental es un reflejo de la necesidad de la industria por eco-innovar y adaptarse a una nueva realidad competitiva.

Se determinó que la eco-innovación aparece como una fuente competitiva clave para el desarrollo sostenible. El turismo, como sector clave y representativo en la economía colombiana no es ajeno a este concepto. Los responsables de la economía en general y del turismo empiezan a manifestar gran interés por este concepto, involucrando por consiguiente líneas de financiación, iniciativas legislativas y trabajos académicos que aporten a la investigación y desarrollo del sector hotelero y su relación con el medio ambiente. 


\section{Referencias}

American Psychological Association (APA). (2010). Manual de estilo Apa. Obtenido de manual de estilo apa según la sexta edición en inglés: http://www.cesdonbosco. com/cii/documentos/Manual_Estilo_APA.pdf

Amaral, S. (4 de Julio de 2007). Natureza bem hospedada. Jornal da comunidade. Obtenido de Especial Meio Ambiente: http://www.empresaresponsavel.com/links/ meio\%20ambiente\%20-\%20jornal\%20da\%20comunidade.pdf. Pagina 4-7.

ANIF. (5 de diciembre de 2011). Comentario Ecónomico del día. Recuperado el 17 de septiembre de 2012, de Dinámica del Sector Turismo en Colombia: http://anif.co/ sites/default/files/uploads/Dic5-11.pdf

Banco de Comercio Exterior de Colombia (BANCOLDEX). (2012). Términos de referencia para la convocatoria nacional de apoyo a la innovación empresarial. Bogotá.

Bayer. (2012). Bayer: Science For A Better Life Brasil. Obtenido de Programa Bayer Jovens Embaixadores Ambientais: http://www.bayerjovens.com.br/

Beni, M. (2003). Globalização do turismo: megatendências do setor e a realidade brasileira. ,. São Paulo: Aleph.

Carrillo, J., González, P., \& Könnölä, T. (2009). Eco-Innovation: When sustainability and Competitiveness Shake Hands. Hampshire: Palgrave Macmillan.

Comisión de Comunidades Europeas. (2001). Fomentando un marco europeo para la responsabilidad social de las empresas. Bruselas.

Comisión de Derechos Humanos del Estado de México (CODHEM). (Noviembre de 2007). Instituto de investigaciones Juridicas de la Universidad Autonoma de México. Recuperado el 12 de Septiembre de 2012, de http://www.juridicas.unam.mx/publica/librev/rev/derhum/ cont/52/pr/pr33.pdf

Comisión Europea. (2004). Developing an Action Plan for Enviromental Technology (ETAP).

Coon, D. (2005). Psicologia. México: Thomson Editores.

Costa, B., \& Sánchez, R. (2006). La empresa sostenible aprendiendo de los líderes del indice Dow Jones de Sostenibilidad (IDJS). Bilbao: Rógar S.A.

Cotelco y Fedesarrollo . (10 de septiembre de 2010). Informe final: Estudio de Prospectiva para la Industria de La Hoteleria. Recuperado el 19 de Septiembre de 2012, de http://www.fedesarrollo.org.co/wp-content/ uploads/2011/08/Estudio-de-prospectiva-para-la-industriade-la-hoteleria-Informe_final-sep-30-2010-definitivo-II.pdf
DANE. (2012). Preguntas Frecuentes. Recuperado el 15 de Septiembre de 2012, de http://www.dane.gov.co/files/ faqs/faq_pib.pdf

Departamento Nacional de Planeación. (2007). Documento CONPES 3527. Bogotá.

Diccionario de la real academia española (RAE) Vigésima edición online. (27 de Junio de 2012). Real Academia Española. Obtenido de Definición de Hotel: http://lema. rae.es/drae/?val=hotel.

Dr. Fernández, R. (2011). La dimensión económica del desarrollo sostenible. Alicante: Editorial Club Universitario.

Eco Hospedagem. (2011). Portal Eco Hospedagem. Obtenido de Lista de hotéis, Pousadas e Resorts sustentáveis: http://ecohospedagem.com/hoteis-pousadas-eresorts-sustentaveis/\#34

Eumed . (25 de Mayo de 2008). Tesis Doctorales . Obtenido de Incentivos al capital humano: http://www.eumed. net/tesis-doctorales/dvt/5.pdf

Europea, C. (2009). Ecoinnovación. Medio Ambiente.

Facultad de Ciencia Administrativas y Contables de la Univerisidad de la Salle. (2010). Currículos Redimensionados. Bogotá: Ediciones Unisalle.

Fernández P, E. (2000). La medición del Impacto Social del la ciencia y la tecnología. Obtenido de http:// www.redhucyt.oas.org/ricyt/interior/biblioteca/polcuch. pdf [Consultado: 9 de noviembre del 2006].

Fernandez, R. (11 de diciembre de 2011). A relação das práticas ambientais e desempenho organizacional na hotelaria de Porto Alegre. Tesis de maestria no publicada. Porto Alegre, Rio Grande Do Sul, Brasil: Universidad Federal Do Rio Grande Do Sul.

Figueredo, Rosa M.; Alcaldia Mayor de Bogotá; Instituto Distrital de Turismo. (2011). Balance Turístico de Bogotá. Obtenido de http://www.revistalabarra.com.co/proyecciones/pdf/memorias/RosaMargaritaFigueredo-IDTenProyecciones.pdf

Fundación EOI, Fundación OPTI. (2012). Green Jobs, empleo verde en España. Valencia, España: Base 12 diseño y comunicación s,l.

Fundación Observatorio de Prospectiva Tecnológica Industria (OPTI) y Fundación EOI Escuela de Organización Industrial. (2010). El marco normativo y competitivo de la economía verde Green jobs. España. 
Fussler, C., \& James, P. (1996). Eco-innovación integrando el medio ambiente en la empresa del futuro. Madrid, España: Mundi-Presa.

Gore, A., \& Blood, D. (14 de diciembre de 2011). A Manifesto for Sustainable Capitalism. The Wall Street Journal, Disponible en: http://online.wsj.com/article/SB10001424 052970203430404577092682864215896.html.

Gore, A., \& Blood, D. (14 de diciembre de 2011). Generation. Obtenido de http://www.generationim.com/media/ pdf-wsj-manifesto-sustainable-capitalism-14-12-11.pdf

Group, T. (2008). Systematic Innovation Panel on ecoinnovation.

Guzzo, R. F. (2011). A relação das praticas ambientais e desempenho organizacional na hotelaria de Porto Alegre. Porto Alegre, Brasil : Universidad Federal Do Rio Grande Do Sul.

Hammel, G., \& Breen, B. (2008). El futuro de la administración. Bogotá: Grupo Editorial Norma.

Hitt, M. (2006). Administración . México: Pearson Prentice Hall.

Hotêlier News. (8 de Octuber de 2009). Noticiero de Hotelería y sustentabilidad de Brasil . Obtenido de Confira as dez redes hoteleiras mais sustentáveis do mundo: http://hoteliernews.com.br/?s=Confira+as+dez+redes+h oteleiras+mais+sustent $\%$ C3\%A1veis+do+mundo

Huppes, K. H. (2008). Measuring Eco-Innovation: Framework And Typology Of Indicators Based On Causal Chains. Final Report Of The Ecodrive Project.

ICONTEC . (2004). Fondo de promoción turística Colombia. Obtenido de Norma técnica sectoria colombiana NTSH 006: http://www.fondodepromocionturistica.com/ docs/documentos/NTSH006.pdf

ICONTEC; Min. De Comercio, Industria y Turismo; Min. De Ambiente, Vivienda y Desarrollo Territorial; Universidad Externado de Colombia. (22 de septiembre de 2006). Norma Técnica Colombiana 5133. Etiquetas ambientales tipo I. Sello ambiental colombiano. Criterios para establecimientos de alojamiento y hospedaje. Bogotá, Colombia: Instituto Colombiano de Normas Técnicas Y Certificación Colombiana (ICONTEC).

Instituto Colombiano de Normas Técnicas y Certificación (ICONTEC); Ministerio De Comercio, Industria y Turismo de la República de Colombia; Ministerio de Ambiente y Desarrollo de la República de Colombia; Universidad Externado de Colombia. (17 de Noviembre de 2006). Norma Técnica Sectorial Colombiana NTS TS 002. Establecimientos de Alojamiento y Hospedaje (EHA). Bogotá, Colombia: Instituto Colombiano de Normas Técnicas y Certificación (ICONTEC).
Instituto Distrital De Turismo. (2012). Turismo sector de alto impacto en Bogotá. Recuperado el 20 de septiembre de 2012, de Plan Integral Mercadeo Turistico: http:// www.investinbogota.org/archivos/anexo5_plan_integral_ mercadeo_turistico.pdf

Jacobs, M. (1997). La Economía Verde: Medio Ambiente, Desarrollo Sostenible y la Política Del Futuro. En M. Jacobs, La Economía Verde: Medio Ambiente, Desarrollo Sostenible y la Política Del Futuro (págs. 115-128). Barcelona: Romanya Valls, S.A.

James, C. F. (1996). Eco-innovación. En C. F. James, Eco-innovación: Integrando el medio ambiente en la empresa del futuro. España: Mundi prensa.

James, C. F. (1996). Eco-innovación integrando el medio ambiente en la empresa del futuro. Madrid, España: Grupo Mundi-Prensa.

Kemp, R. a. (2008). Final report MEI project about measuring eco-innovation.

Maketing Publishing Center. (1990). Instrumentos de análisis de márketing estratégico. Madrid, España: Ediciones Díaz de Santos, S.A.

Millán, N., Rosa, M., \& Villarroel, M. (septiembre de 2009). Trabajo Final para la obtención del título de Master en Gerencia y Administración Universidad de la Republica. Responsabilidad Social y Medio Ambiente El Rol del Estado. Montevideo, Uruguay.

Min de Ambiente, Vivienda y Desarrollo Territorial CoIombiano; ICONTEC. (06 de junio de 2006). SELLO AMBIENTAL COLOMBIANO. Selección y normalización de categorías de producto para el sello ambiental colombiano. Grupo de Mercados Verdes. Bogotá, Colombia: Ministerio de Ambiente, Vivienda y Desarrollo Territorial e ICONTEC.

Ministerio de Comercio, Industria y Turismo. (2002). Programa de Transformación Productiva (PTP). Bogotá.

Nogales, Á. F. (2004). Investigación y técnicas de mercado. Madrid: Esic editorial.

Observatorio Turístico de Bogotá. (mayo de 2011). Boletin No. 5 - Mayo de 2011. Recuperado el 19 de septiembre de 2012, de http://www.bogotaturismo.gov.co/sites/ default/files/archivo/08/boletin_05-2011.pdf

Oficina de Prensa DANE. (22 de marzo de 2012). Comunicado de prensa: Producto Interno Bruto - Cuatro Trimestre y Total Anual 2011. Recuperado el 10 de Septiembre de 2012, de http://www.dane.gov.co/files/investigaciones/boletines/pib/cp_PIB_IVtrim11.pdf 
OMT. (2011). Panorama OMT del Turismo Internacional Edición 2011. Recuperado el 16 de septiembre de 2012, de http://mkt.unwto.org/sites/all/files/docpdf/unwtohighlights11sphr_1.pdf

OMT. (16 de enero de 2012). UNWTO Organización Mundial del Turismo OMT. Recuperado el 15 de Septiembre de 2012, de Comunicado de prensa:El turismo internacional alcanzará la cifra de los mil millones en 2012: http://media.unwto.org/es/press-release/2012-01-16/ el-turismo-internacional-alcanzara-la-cifra-de-los-milmillones-en-2012

Organización para la Cooperación y el Desarrollo Económico. (2008). Sustainable manufacturing and eco-innovation: First steps in building a common analytical fraemwork.

Pampillón, R. (22 de octubre de 2009). Blog: Diccionario de economía, energía, medio ambiente y cambio climatico. Recuperado el 2 de Julio de 2012, de $i$ Qué es la eco-innovación?: http:/economy.blogs.ie.edu/ archives/2009/10/\%C2BFque-es-la-eco-innovacion.php

Pérez, W. (noviembre de 2012). Trabajo de grado para optar al título de especialización en la Universidad Externado De Colombia. Diseño de metodología y herramientas para la innovación disruptiva de modelos de negocios aplicable a cualquier emprendimiento o empresa existente. Bogotá, Colombia.

Periodico Online de la Cámara de Valencia . (12 de marzo de 2012). Cada Vez Más Importante Para La Economía Mundial. Recuperado el 10 de Septiembre de 2012, de http://www.camaravalencia.com/camaraonline/contenidoNoticia.asp? noticia $=20120312085202$

Petrobras S.A. (2011). Petrobras Relatorio de sostenibilidad . Obtenido de informe de sosteniblidad 2010: http:// www.petrobras.com.br/rs2010/es/relatorio-de-sustentabilidade/resultados-contribuicoes-sociedade/contribuicoessociedade-impactos-indiretos/investimentos-sociais/

Plan Nacional de Desarrollo. (2010-2014, Pág 56). Conocimiento e Innovación. Bogotá.

PNUMA. (2011). Hacia una economía verde. Obtenido de Guía para el desarrollo sostenible y la erradicación de la pobreza. Sintesís para los encargados de la formulación de políticas: http://www.unep.org/greeneconomy/ Portals/88/documents/ger/GER_synthesis_sp.pdf

Porter, M. (1982). Estrategia competitiva. Técnica para el Análisis de los Sectores Industriales y de la Competencia.. 1982, $1^{\text {a }}$. Edición. P. 61-62. (Primera Edición ed.). México: Editorial CECSA.

Porter, M. (2009). On competitive (Actualizada 2009 ed.). Barcelona: Harvard Business Review.
PROEXPORT COLOMBIA. (Enero de 2012). Informe de Turismo Extranjero en Colombia a 2011. Recuperado el 18 de Septiembre de 2012, de http://www.serconatural. com/assets/informe-dic-turismo-extranjero-en-colombia-a-d.pdf

PROPAIS . (22 de febrero de 2012). Corporación para el desarrollo de las microempresas. Diagnóstico de servicios de desarrollo empresarial en Colombia: fortalezas, retos y oportunidades.

Ríos Ortiz de Montellano, M. B. (2004). Colección de Tesis Digitales Universidad de las Américas Puebla (CIRIA UDLAP). Obtenido de Del mesón al hotel en la Puebla de los Ángeles: catarina.udlap.mx/u_dl_a/tales/ documentos/lhr/rios_o_mb/capitulo2.pdf

Secretaria de Turismo . (15 de julio de 2010). Programa Sectorial de Turismo 2010 - 2015. Periódico Oficial del Estado Libre y Soberano de San Luis de Potosí, pág. 6.

The Coca-Cola Company. (2011). Coca-Cola Brasil . Obtenido de COCA-COLA BRASIL NO DIA MUNDIAL DO MEIO AMBIENTE: http://www.cocacolabrasil.com.br/release_detalhe.asp? release $=51 \&$ Categoria $=35$

UNEP (Programa de las Naciones Unidas para el Medio Ambiente). (2012). XVIII Reunión del Foro de Ministros de Medio Ambiente. Obtenido de Economía Verde en el contexto del Desarrollo Sostenible y erradicación de la pobreza: una perspectiva desde América Latina y el Caribe.: http://www.pnuma.org/forodeministros/18-ecuador/Reunion\%20Expertos/Informe\%20Economia\%20 Verde/ESPANOL\%20Economia \%20Verde\%2016\%20 DEC\%202011.pdf

Universidad de Burgos. (Noviembre de 2010). La Responsabilidad Social en las Universidades Españolas 2010. Burgos, Provincia de Burgos, España.

Universidad del Rosario. (2011). Turbulencia empresarial en Colombia:el caso del sector hotelero. Documentos de Investigación: Facultad de Administración. Bogotá: Universidad del Rosario.

Universidad Santiago de Cali. (2008). Responsabilidad Social Empresarial: Su origen, evolución y desarrollo en Colombia. Obtenido de Redunirse Org: http://redunirse.org/nuevo/sites/default/files/pdf/Responsabilidad\%20Social\%20 Empresarial\%20su\%20origen,\%20evoluci\%C3\%B3n\%20 y\%20desarrollo\%20en\%20Colombia.pdf

Valencia, J. (2012). Boletin turístico. Obtenido de Diccionario de términos turísticos: http://www.boletin-turistico. com/diccionarioturismo. 